(Aus dem deutschen physiologischen Institut zu Prag.)

\title{
Die Wirkung der physiologischen Kochsalzlösung auf quergestreifte Muskeln.
}

Von.

F. S. Locke aus Cambridge.

Hierzu Tafel V.

Nachdem Kölliker die „Indifferenz" der Lösungen des Chlornatrium von $0,5-1$ p. Ct. in Wasser gefunden hat, $d$. h. die Thatsache, dass sie z. B. mit den Muskeln lange in Berubrung bleiben können, ohne dass letztere ihre Erregbarkeit verlieren, sind diese Lösungen allgemein gebraucht, um physiologische Präparate besonders von Nerven und Muskeln feucht zu erhalten, oder das Blut aus den Gefässen auszuspritzen. Die Thatsache, dass Muskeln in diesen Lösungen ihre Erregbarkeit erhalten, scheint als genügender Beweis dafür angesehen worden zu sein, dass sie keinen merklichen Einfluss auf die Eigenschaften von Muskeln haben, und dass ihr Gebrauch keine störenden Veränderungen mit sich bringt.

Erst in den letzten Jahren wurde durch die später zu besprechenden Untersuchungen diese Auffassung erschüttert. In wie mannigfacher Weise aber der Gebrauch solcher Lösungen bei Muskeluntersuchungen sehr störende Folgen bedingen kann, ist noch nicht dargelegt. Die Erörterung dieser Folgen ist der Gegenstand der vorliegenden Abhandlung, welche leider vorzeitig abgebrochen werden musste, und daher nicht den Anspruch auf irgend erschöpfende Behandlung ihres Gegenstandes macht.

I.

Im April 1891 sah ich mich gelegentlich veranlasst, in einem Controlversuche die Grösse der nach Momentanreiz erfolgenden negativen Schwankung bei Muskeln, welche in 0,6 procentige Kochsalzlösung (K.-S.-L.) durch eine halbe Stunde oder mehr gelegt worden waren (Kochsalzmuskeln), und bei solehen, die nicht in der Lösung gelegen hatten, vergleichsweise zu bestimmen. Ich war 
überrascht, einen grossen Unterschied zu finden: die negativen Schwankungen eines Sartorius, der in K.-S.-L. gelegen hatte, waren viel grösser als diejenigen des andern Muskels, der entweder in der feuchten Kammer aufbewahrt oder frisch untersucht worden war.

In den vielen Versuchen, welche ich machte, war dieses Resultat ganz constant: zuweilen war der Unterschied nicht sehr gross, aber immer ganz deutlich.

Ich gebe zwei Protocolle als Beispiele.

Das untere Ende des Sartorius wurde mit einem erhitzten Glasstäbchen abgetödtet. Die eine Boussolelectrode wurde an dieses Ende, die andere ungefähr an die Mitte des Muskels angelegt. Die Platinelectroden zur Reizung wurden nahe dem oberen Ende angelegt. Verwendet wurde das Herm a n n'sche Galvanometer.

Nr. I. 18. April 1891. Rana temporaria. Curarisirter Sartorius, $35 \mathrm{Mi}$ nuten in K. S. L. von $0,6 \%$ gelegt.

Electrodenstrom $=-9$ sc. Muskelstrom in Compens. -Graden $=609$. Rollenabstand des Inductoriums $=4,7 \mathrm{~cm}, 1$ Daniell im primären Kreise.

S. S. = Schliessungsinductionsschlag.

Oe. $\mathrm{S}_{\mathrm{s}}=$ Oeffnungsinductionsschlag. bedeutet langsame Zunahme des Muskelstromes.

S. S. $\quad-8,25$

Oe. S. $\quad-82$

S. S. $\quad-6$

Oe. S. -44

S. S. $\quad-6$

Oe. S. $\quad-16$

S. S. $\quad-5$

Oe.S. $-4,5$

S. S. -4

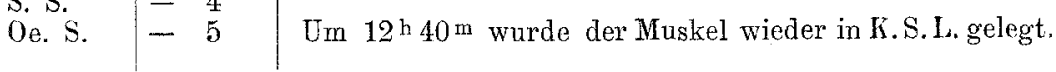

Der andere Sartorius, der durch 70 Minuten in der feuchten Kammer gelegen hatte.

Muskelstrom in Comp.-Graden $553<$.

\begin{tabular}{l|l}
\hline S. S. & -4 \\
Oe. S. & $-4,5$ \\
S. S. & $-\quad 3$ \\
Oe. S. & $-3,5$ \\
S. S. & $-2,5$ \\
Oe. S. & $-3,5$ \\
S. S. & $-2,5$ \\
Oe. S. & -3
\end{tabular}

Zwischendurch noch acht Oeffnungsinductionsschläge durch den Muskel geschickt.

Um $1^{\mathrm{h}} 10^{\mathrm{m}}$ wird der Muskel in dieselbe Lösung wie der erste Muskel gelegt. Um $3^{h}$ wieder untersucht. Muskelstrom in Comp. Graden $490<$. 
Die Wirkung d. physiologischen Kochsalzlösung auf quergestreifte Muskein. 503

\begin{tabular}{l|l}
\hline & \\
Oe. S. & -4 \\
S. S. & -2 \\
Oe.s. & -3 \\
S. S. & $-1,75$ \\
Oe. S. & -2 \\
S. S. & -1 \\
Oe. S. & -2 \\
S. S. & $-1,75$
\end{tabular}

Um 3 h $20 \mathrm{~m}$ wurde der erste Muskel wieder untersucht.

Muskelstrom in Comp.-Graden 459.

\begin{tabular}{l|l|l}
\hline \hline S. S. & - & 2,5 \\
Oe. S. & - & 3 \\
Oe. S. & - & 3
\end{tabular} \mid Secundäre Spirale bis 1,7 cm Abstand verschoben.

Nr. II. 15. April 1891. Rana temporaria. Frischer curarisirter Sartorius.

E. S. -1 sc. Muskelstrom in Comp.-Gr. $=378<$. Rollenabstand $=4, \pi \mathrm{cm}$.

S. S. $\quad-2,75$

Oe.S. $\quad-\quad 2,75$

S. S. $\quad-2,75$

Oe. S. $-2,75$

Der andere Sartorius war durch 2 Stunden 40 Min. in K. S. L. gewesen. Muskelstrom in Comp.-Graden $420<$.

S. S. $\quad-21$

Oe. S. -52

S. S. -14

Oe. S. $\quad-26$

oe. S. $\quad-15$

Bemerkenswerth ist die relativ viel grössere Wirksamkeit des Oeffungsschlages und die mit jeder neuen Reizung eintretende Verminderung der negativen Schwankung. Ich unterliess es zunächst, besonders zu untersuchen, ob Ruhe des Muskels eine Rückkehr der grossen Schwankungen mit sich bringt. Aus später zu erwähnenden Gründen hielt ich dies für überflüssig.

Nachdem ich von der Constanz dieser Erscheinung überzeugt war, ging ich daran zu untersuchen, ob K.-S.-L. von $0,6 \%$ eine entsprechende Wirkung auf die Muskelcontraction hat.

II.

Es ist lange bekannt, dass die Natriumsalze einen Einfluss 
auf die Muskelcontraction haben. O. Nasse ${ }^{1}$ ) beobachtete einen tetanischen Verlauf statt einer Zuckung, wenn der Muskel mit einer Lösung ron Natriumsulfat oder -carbonat behandelt worden war, was Biederma n $n^{2}$ ) gelegentlich bestätigen konnte. Brunton und $\mathrm{Cash}^{3}$ ) fanden von Chlornatrium, dass napplied locally of the strength of 0,7 per cent there appears to be no active change in the curve (This is therefore rightly called normal salt solution)". Sie arbeiteten mit einem dicken Muskel (Gastrocnemius). Ringer hat den Einfluss der $0,6 \%$ K.S.S.L. auf quergestreifte Muskeln zu bestimmen gesucht, indem er das eine Mal in solcher Lösung befindliche Sartorii in knrzen Intervallen reizte ${ }^{4}$ ), das anderemal Gastroenemii von Fröschen, die mit der Lösung durchströmt wurden, untersuchte ${ }^{5}$ ). Letzteres hat auch Carslaw gemacht $\left.{ }^{6}\right)$. Ringer fand eine contracturfördernde Wirkung der K.-S.-L. und erwähnt in seiner Abhandlung uber die Perfusionswirkung der K.-S.-L.: „While the rate of contraction is slowed the period of relaxation is often enormously prolonged, and these changes are constant until contractility has quite disappeared. In some experiments we found that soon after the commencement of perfusion the amount of contraction, as shown by the height of the trace, is greatly inereased, although the strength of the excitation remained the sames"

Carslaw arbeitete mit Lösungen, welche $0,5 \% \mathrm{NaCl}$ und dariiber enthielten, und fand: "ein Muskel, der von den genannten Lösungen viele Minuten lang durchflossen war, führt, wenn ein Inductionsschlag ihn reizbar findet, Zuckungen aus, die mit einer sogenannten Contractur behaftet sind. Die Contractur ist entweder die einfache, wie sie von $\mathrm{Kronecker}$ und Tiegel beschrieben wurde, oder es gleicht die Curve der Zuckung der eines dicrotischen Pulses, indem die auf die erste Erbebung folgende Senkung von einem neuen Ansteigen unterbrochen wird." Carslaw scheint keine Frscheinungen, die auf gesteigerte Erregbarkeit der Muskeln deuten, gesehen zu haben. Bei meinen Versuchen liess ich Sartorien, die in 0,6\% K.-S.-L. gelegen hatten,

1) Dieses Archiv II. S. 117.

2) Sitz.-Ber. der Wiener Akad. LXXX. Bd. III. Abth. S. 388.

3) Philos. Transact. 1884. S. 226.

4) Journ. of Physiol. VIII. S. 20.

5) Ebendas. VIII. S. 288.

6) Du Bois Rey mon d's Archiv. 1887. S. 429. 
ihre Zuckungen aufschreiben. Die Muskeln waren noch in Verbindung mit den Knochenstïmpfen. Die Inductionsströme wurden meist durch den ganzen Muskel, zuweilen auch durch zwei an den Verlauf des Muskels gelegte Platinelectroden geschickt und waren von derselben Stärke, wie bei meinen galvanometrischen Versuchen. Ganz in Uebereinstimmung mit letzteren zeigten sich sogleich bei den ersten Versuchen nicht einfache Zuckungen, sondern tetaniforme Contractionen von enormer Höhe und einer. Dauer von mehreren Secunden, nach welchen der Muskel plötzlich erschlaffte und nur einen kleinen Verkürzungsrückstand zeigte. Weitere Versuche lehrten dann, dass diese eigenthitmlichen Contractionen erst bei grösseren Reizstärken und, in Uebereinstimmung mit den galvanometrischen Erscheinungen, besonders beiOeffnungs-lnductionsströmen (Oe.-I..Strömen) auftraten. Wurde ein solcher Muskel zunächst mit den schwächsten, eben wirksamen Strömen gereizt, and durch Annäherung der Spiralen der Reiz stufenweise verstärkt, so waren die ersten Zuckungen stets normal; bei einem Rollenabstand von 4-5 cm (1 Daniell) schloss sich an die durch den Oe.I.-Strom bewirkte Zuckung ein Verkürzungsrückstand an, der sich bei noch stärkeren Reizen vergrösserte; erst als die Rollen über einander geschoben waren, trat bei Reizung dureh den Oe.-I. Strom die tetanische Contraction auf (Fig. 1), jedoch nur einmal in voller Stärke. Wurden die Reizungen fortgesetzt, so verlor sich die tetanische Contraction wieder, und nach einem nochmaligen Stadium, in welchem sich lediglich ein Verkürzungsrückstand nach der Zuckung zeigte, gab der Muskel wieder nur noch einfache Zuckungen. Dies entspricht dem allgemeinen Verhalten der "Contractur“.

In solcher Weise verhielten sich-Muskeln, welche ganz frisch oder nach nur wenigen vorläufigen Controlreizungen eine halbe Stunde oder länger in der K.-S.-L. gelegen hatten.

Wurde aber ein frischer Maskel häufig gereizt und dann eine halbe Stunde in die K.-S.-L. gelegt, oder wurde ein schon einmal in dieser Lösung gewesener und darnach hänfig gereizter Muskel zum zweitenmale der halbstündigen Einwirkung der Kochsalzlösung ausgesetzt, so zeigte sich nachher an solchen Muskeln zwar auch die überwiegende Wirksamkeit der Oe.-I.-Ströme und es traten auch hier und zwar wieder erst bei genügender Reizstärke eigenthümliche Contractionen auf, aber dieselben waren nicht einfach tetaniform, sondern zweigipfelig, ähnlich der „Doppelzuckung“" 
eines veratrinisirten Muskels. Die Erschlaffung nach der ersten Zuckung war sehr ausgeprägt und erreichte bisweilen wieder die Abscisse; die zweite Zusammenziehung war etwas verzögert und konnte die erste Zuckung übertreffen oder nicht. Die auf diese zweite Contraction folgende Erschlaffung verlief viel schneller als am veratrinisirten Muskel und war zuweilen durch Verzögerungen oder nochmalige Zusammenziehungen unterbrochen, die gelegentlich die Höhe ihrer Vorgänger übertrafen. Bei Wiederholung der Reizung verschwanden diese eigenthümlichen Contractionserscheinungen wieder ebenso, wie im erstbescbriebenen Falle die rein tetaniformen Contractionen.

Die beschriebenen vorläufigen Versuche wurden in einer Woche des April angestellt, und ich war noch mit den Vorbereitungen zu einer eingehenden Untersuchung der in theoretischer und methodischer Hinsicht interessant scheinenden Thatsachen beschäftigt, als auf das bisher kithle Wetter plötzlich warme Witterung folgte.

Von da $a b$ waren die Erscheinungen dahin geändert, dass die Einwirkung der K.-S.-L. nur einen einfacben Verküirzungsriuckstand zur Folge hatte. Die Muskeln der Frösche und zwar sowohl der im Institut gehaltenen, als auch der frisch eingefangenen, hatten nicht mehr jene schöne rothe Farbe, die das Zeichen ihres guten Zustandes ist, bei welchem allein diese Wirkung der K.-S.-L. zu beobachten ist.

Die Frösche, womit ich gearbeitet hatte, waren nicht überwintert, sondern im Frühling eingefangen.

Erst Ende Mai und Anfang Juni konnte ich die Versuche fortsetzen und sah auch wieder gelegentlich tetaniforme Contractionen. Das Wetter war relativ kühl, die Muskeln schön roth und in sehr gutem Zustande. Die Folgen der Einwirkung der K.-S.-L. aber waren nicht ganz dieselben wie im April. Der Unterschied in der Wirkung der S.-I.-Ströme und Oe.-I.-Ströme war nicht so ausgeprägt. Häufig sah man ganz schwache Schläge sehr verlängerte Contractionen veranlassen, doch ohne bestimmte Regel (s. Fig. 6). Auch war der Einfluss der vorhergehenden Reizungen nicht so ausgeprägt. Nach wiederbolten Reizungen bekam man immer noch verlängerte Contractionen: es gab nur eine Verminderung ihrer Höhe (s. Fig. 8). Bei höherer Aussentemperatur scheint sonach die Wirkung der K.-S.-L. eine grössere zu sein, wie dies 
bekanntlich bei Veratrin der Fall ist. Ich sah jetzt auch nicht so ausgeprägte zweigipfelige Curven wie im Frühling. Die Contractionen waren beinahe immer einfach tetaniform, aber von verschiedener Daver und Erschlaffungsschnelligkeit, oder verlängerte Zuckungen. Àm häufigsten schloss sich unmittelbar an die Zuckung. eine Contractur von grosser Höhe an, ohne eine zwischenliegende Einsenkung der Curve (s. Fig. 4, 6, 8 u. 9).

Die Regelmässigkeit einer Erscheinung, welche ich im Frühling gesehen, aber nicht besonders untersucht hatte, konnte ich jetzt vollkommen nachweisen. Wenn nach wiederholten Reizungen die Contractionen eines Kochsalzmuskels sich sehr vermindert haben, erreichen sie nach Rube (10 Minuten oder mehr) in K.-S.-L. beinahe oder vollkommen wieder ihre frühere Grösse. Diese „Erbolung" konnte mehrmals bei demselben Muskel vorkommen. Sie erinnert wieder an den veratrinisirten Muskel (s. Fig. 9).

Der Einfluss der Stromesrichtung auf die Folgen der Reizung ist häufig bei Kochsalzmuskeln besonders stark ausgeprägt und zwar nicht nur bei schwachen, sondern auch bei starken Reizen.

Wie man schon nach dem Vorigen erwarten kann, wird die Erregbarkeit des Muskels durch K.-S.-L. gesteigert. Dies zeigt sich beim Vergleich der Minimalreize vor und nach der Einwirkung der K.-S.-L. und auch beim Vergleich der Zuckungshöhen. Ueber den Einfluss von K.-S.-L. auf Muskelerregbarkeit werde ich später mehr zu sagen haben.

Auffallend ist die Leichtigkeit, womit man einen Kochsalzmuskel wieder normale Zuckungen geben lassen kann. Ringer hat gefunden, dass die Hinzusetzung von einem Theil $\mathrm{CaCl}_{2}$ zu 5000 Theilen der K.-S.-L., in welcher der Muskel gereizt wird, den Verkürrungsrïckstand, den er unter diesen Umständen beobachtete, vollkommen aufhebt. Dadurch angeregt, versuchte ich die Wirkung einer K.-S.-L., die 10 Procent einer gesättigten Lösung von $\mathrm{CaSO}_{4}$ enthält, auf einen Kochsalzmuskel, der ganz ausgeprägt die Wirkung der K.-S.-L. zeigte. Nach 5 Minuten Eintauchung gab der Muskel mit allen Reizstärken nur einfache Zuckungen, nach welchen aber die Erschlaffung nicht sogleich vollkommen war; es blieb ein kleiner Verkürzungsrückstand, welcher nach längerer Eintauchung in der Ca-Lösung noch vorhanden ist. Nichts destoweniger ist die Wirkung von Ca sehr auffallend und ganz constant (s. Fig. 4 und 5). Taucht man einen mit $\mathrm{CaSO}_{4}$-K.-S.-L. 
behandelten Muskel für eine halbe Stunde oder mehr in K.-S.-L. ein, so kehren die tetaniformen Contractionen zuriek und können wieder durch $\mathrm{CaSO}_{4}$ beseitigt werden.

Ich habe nicht weiter den Einfluss versehieden starker Zusätze von Ca-Salzen zur K.-S.-L, untersucht. Es scheint aber fast, als ob eine $\mathrm{CaSO}_{4}$-haltige K.-S.-L. mehr „physiologisch" ist, als eine reine K.-S.-L. Hier möchte ich auch erwähnen, dass, soweit ich gelegentlich beobachtet habe, ein Sartorius in der $\mathrm{CaSO}_{4}$-haltigen K.-S.-L. nicht „spontan“ zuckt. Diesen Einfluss von Ca-Salzen hat schon Ringer gefunden.

Dies sind meine vorläufigen Ergebnisse, die noch mannigfache Ergänzung fordern. Inbesondere wäre noch näher zu untersuchen die Elasticität des Muskels während der langdanernden Contractionen, der Einfluss der Erwärmung und Abkühlung des Kochsalzmuskels auf seine Contractionen, die Wirkung der Kettenströme, bezïglich welcher ich vorläufig sagen kann, dass der Strom von 2 Dasiell eine langdauernde Contraction auslösen kann. Anch das Verbalten eines Kochsalzmuskels bei indirekter Reizung ist noch nicht genügend untersucht. Ob ein Kochsalzmuskel in dieser Beziehung dem veratrinisirten Muskel gleicht, weiss ich nicht. Es sei erwähnt, dass Tie g e ${ }^{1}$ ) bei indirecter Reizung eine Contractur nicht beobachten konnte, obgleich er Frösche, deren Gefässe mit K.-S.-L. ausgespritzt worden waren, benitzte. Die Thatsache aber, dass er auch bei noch bestehender Circulation Contractur ebenso wie bei Salzfröschen fand, scheint bei den von ihm benützten Fröschen eine Wirkung der K.-S.-L. auszuschliesseu. Nichts destoweniger kann kaum davon abgesehen werden, dass die zwei Forscher, die zuerst "Abscissenerhebung“" oder "Contractur" klar erkannten, Kronecker. und Tiegel, in ibren Versuchen Salzfrösche benïtzten.

Es ist merkwürdig, dass Niemand, Ringer ausgenommen, and er nur gelegentlich beim Perfundiren der Muskeln, die von K.-S.-L. verursachten tetaniformen Contractionen vọ grosser Höhe gesehen hat. Ich bin iberzengt, dass gute rothe Muskeln sie immer zeigen. Dass Ringer dieselben bei Sartorien, die gleich nach dem Eintauchen in K.-S.-L. regelmässig gereizt wurden, nicht beobachtete, hing wohl davon ab, dass die vor dem Eindringen der

1) Dieses Archiv. XIII, S. 71. 
K.-S.-L. erfolgten Reizungen selbst die Folgen der Wirkung der K.-S.-L. aufhoben; und vielleicht waren auch seine Muskeln nicht gut. In meinen Versuchen, habe ich die Muskeln nicht weniger als 10 Minuten in K.-S. L. gelassen, ehe ich sie reizte. Einmal aber beobachtete ich bei einem Muskel, der, nachdem er etwas trocken geworden war, nur dreimal mit K.-S.-L. gut gepinselt wurde, ihre ausgeprägte Wirkung.

Ich muss hinzufügen, dass die in meinen Versuchen allgemein beniitzte K.S. L. mit Steinsalz gemacht war, und dass die Resultate mit einer chemisch reinen Lösnng controlirt wurden.

Ich habe nur mit Rana temporaria gearbeitet.

\section{III.}

Eine der im letzten Abschnitte beschriebenen Folgen der Einwirkung der K.-S.-L. auf quergestreifte Muskeln ist die Erböhung der Erregbarkeit. Diese Wirkung kann in gewissen Versuchen sehr eigenthümliche Resultate herbeiführen und zwar gerade bei Muskeln, bei denen sich die oben beschriebenen Einflisse der K.-S.-L. auf die Contraction noch garnicht zeigen. Gerade deshalb darf diese Wirkung nicht vernachlässigt werden, wie die folgenden Erörterungen zeigen.

Versucht man den bekannten Einfluss der Stromesrichtung auf die Erregung eines Sartorins dadurch zu beseitigen, dass man den Muskel in ein mit K.S.-L. gefülltes Rohr einführt, um die Stromfäden im Muskel möglichst parallel zu machen und die grössere Stromdichte am untern Muskelende zu beseitigen,' so zeigt sich jener Einfluss nicht aufgehoben. Nachdem ich diese zunächst auffallende Erscheinung beobachtet hatte, fand ich, dass Leicher ${ }^{1}$ ). sie schon gesehen hat, und die Ursache davon „in den unregelmässigen Muskelenden" sucht: „an dem tibialen Muskelende treten die Stromfäden in grösserer Ausdehnung aus dem natürlichen Längsschnitt des Muskels aus, als an dem Beckenende." Ich muss sagen, dass ich diese Erklärung nicht verstehen kann, und daher eine andere in dem Erregbarkeit steigernden Einflusse der K.-S.-L. gesucht habe.

Darauf wurde ich durch die Beobachtung geleitet, dass der Einfluss der Stromesrichtung, statt nach Einfülurung der K.-S.-L. in das Rohr aufgehoben zu werden, trotz der jetzt kleineren

1) Untersuchungen aus d. physiol. Inst. in Halle. H. I. S. 20. 
Stromesdichte am untern Muskelende sogar sehr vergrössert erschien. Die Erklärung dieser Thatsache kann sich nur darin finden, dass die Steigerung der Erregbarkeit dureh die K.-S.-L. sich leichter am dünneren untern Muskelende entwickelt.

Ist diese Auffassung richtig, so muss man das umgekehrte Verbältniss beobachten, wenn statt der K.-S.-L. eine Erregbarkeitherabsetzende Flüssigkeit benutzt wird. Darum stellte ich Versuche mit Lösungen von Kalisalzen an, die bekanntlich diese Eigenschaft besitzen, und fand nach vielen Versuchen eine 0,1 procentige KCl-Lösung am geeignetsten für diesen Zweck; stärkere Lösangen wirken zu stark chemisch erregend.

Wenn ein Sartorius, der stärker bei absteigenden als bei aufsteigenden Inductionsströmen zuckt, in eine 0,1 procentige Lösung von $\mathrm{KCl}$ für kurze Zeit (zwei oder mehr Minuten) eingetaucht wird, so findet man nach Entfernung der Lösung das umgekehrte Verhältniss und zwar entweder sogleich, oder wenn die Lösung nicht lange eingewirkt hat, nach einigen Minuten, weil die Kaliwirkung sich nach Entfernung der KCl-Lösung fortsetzt.

Beispiele dieser Wirkung einer 0,1 procentigen KCl-Lösung geben die folgenden Protocolle.

Nr. III. 20. Mai 1891. Rana temporaria, curarisirter Sartorius mit $3 \mathrm{gr}$ Belastung (die wahre Spannung des Muskels betrug nur 0,35 davon). 5 Daniellsche Elemente im primüren Krreise (so viele wurden benützt, um einen trägen Reizmerker arbeiten zu lassen) ${ }^{1}$ ).

\begin{tabular}{|c|c|c|c|c|c|}
\hline 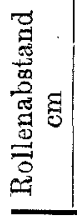 & 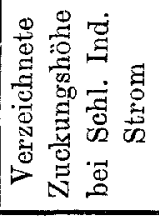 & 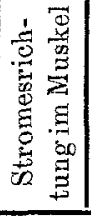 & 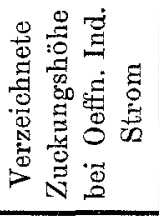 & 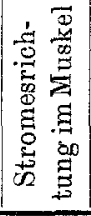 & Bemerkungen \\
\hline $\begin{array}{l}6,5 \\
6,5 \\
6,5 \\
6,5 \\
6 \\
6 \\
6 \\
6\end{array}$ & $\begin{array}{l}5,5 \\
0 \\
6 \\
0 \\
0 \\
0 \\
9 \\
9\end{array}$ & $\downarrow$ & $\begin{array}{c}0,5 \\
9,5 \\
1 \\
9 \\
10 \\
11 \\
6 \\
6\end{array}$ & $\begin{array}{l}\uparrow \\
\downarrow \\
\downarrow \\
\downarrow \\
\downarrow \\
\uparrow\end{array}$ & $\begin{array}{l}\text { frischer Muskel in der Luft ge- } \\
\text { reizt. } \\
\text { Jetzt wurde der Muskel durch } \\
2 \text { Min. 45 Sec. in KCl-Lösung ein- } \\
\text { getaucht. }\end{array}$ \\
\hline
\end{tabular}

1) Die im Allgemeinen unzweckmässige Einschaltung eines Elektromagneten in den primären Kreis erschien hier unbedenklich. 
Die Wirkung d. physiologischen Kochsalzlösung auf quergestreifte Muskeln. 511

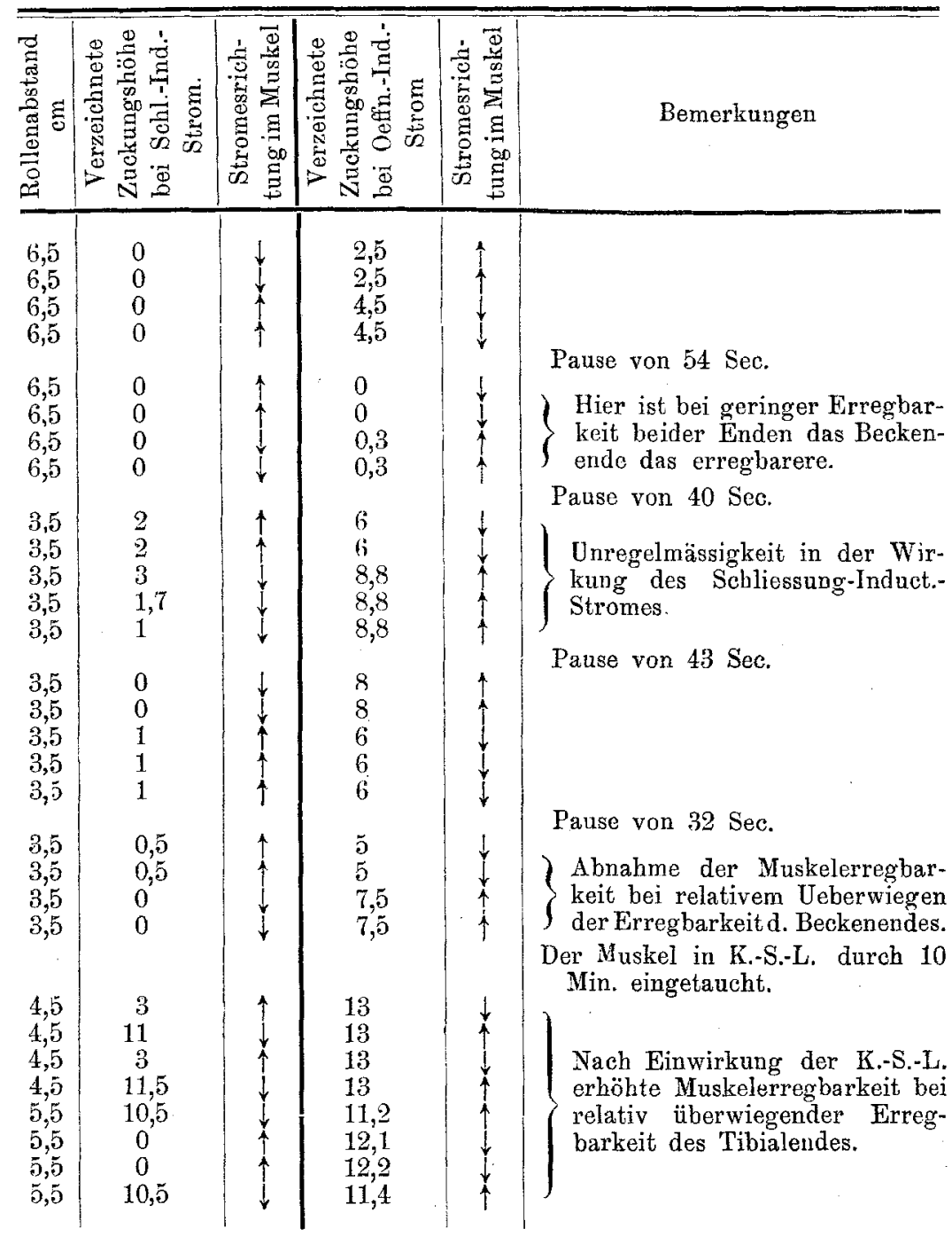

Nr. IV. 26. Mai 1891. Alles wie beim vorigen Versuche.

\begin{tabular}{|c|c|c|c|c|c|}
\hline 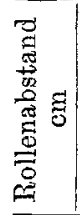 & 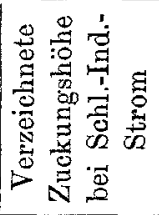 & 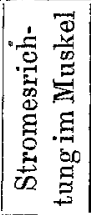 & 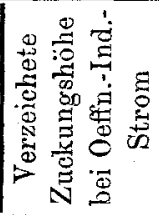 & 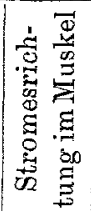 & Bemerkungen \\
\hline $\begin{array}{l}8 \\
8 \\
8 \\
8\end{array}$ & $\begin{array}{l}0 \\
0 \\
6,5 \\
6,5\end{array}$ & $\downarrow$ & $\begin{array}{l}8 \\
8 \\
0,5 \\
0,5\end{array}$ & $\downarrow$ & $\begin{array}{l}\text { Frischer Muskel in der Luft } \\
\text { gereizt } \\
\text { Muskel durch } 4 \text { Min. 25 Sek. in } \\
\text { KCl-Lösung eingetaucht }\end{array}$ \\
\hline
\end{tabular}




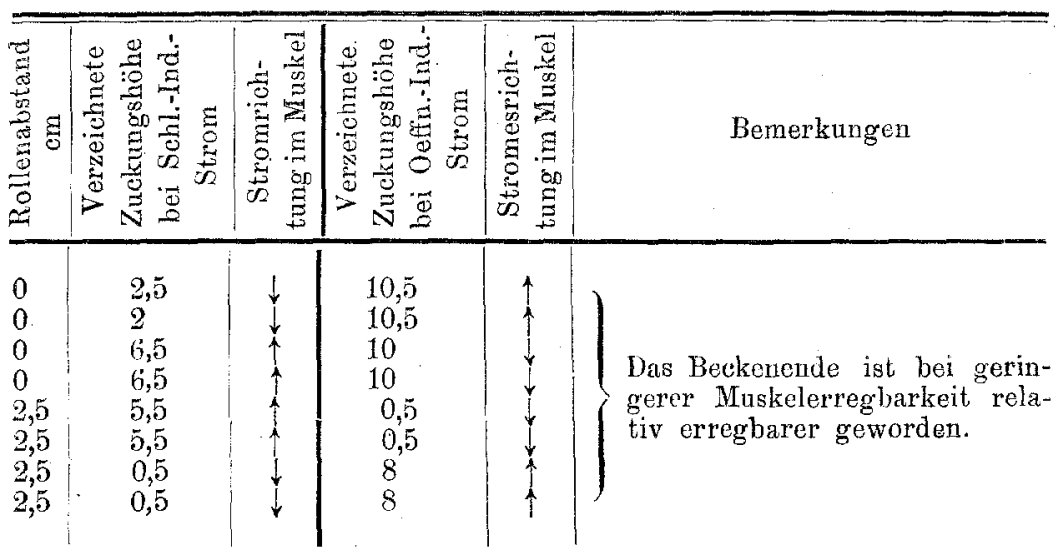

Interessant ist zu sehen, wie die KCl-Wirkung durch eine mehrere Minuten lange Eintauchung des Muskels in K.-S.-L. aufgehoben wird und die normale stärkere Wirkung der absteigenden Ströme zurückkebrt. Es scheint mir, dass die beschriebenen Erscheinungen ibre Ursache nur darin finden können, dass das untere Ende des Sartorius leichter von Fliissigkeiten angegriffen wird, als das abere. Wenn also K.-S.-L. Erregbarkeit-erhöhend wirkt, kann man ganz gut verstehen, warum in Leicher's und in meinen Versuchen die Reizung des Muskels in einem Bette von K.-S.-L. nicht die Gleichheit der Wirksamkeit der aufsteigenden und absteigenden Ströme zur Folge hat.

Um die Erregbarkeit-erböhende Wirkung der K.-S.-L. direkt zu beweisen, habe ich so schnell wie möglich präparirte Sartorii in das leere Glasrohr eingeführt, und nach einigen vorläufigen Reiznngen nur das untere Ende des Rohres, welches das Beckenende des Muskels enthielt, durch ein Ansatzrohr mit K.-S.-L. gefiullt. Nach einer gewissen Zeit wurde die K.-S.-L. abgezogen und die Erregbarkeit des Muskels wieder geprüft. Die Ergebnisse dieser Versuche waren in mehr als einer Beziehung interessant.

Wenn man den. mässig belasteten Muskel bei einem solchen Rollenabstande reizt, dass aufsteigende Oeffnungsschläge nur minimale Zuckungen hervorrufen, wobei die Zuckungen bei umgekehrter Stromesrichtung natürlich ziemlich gross sind, und dann für ein paar Minuten K.-S.-L. am obern Ende des Muskels applicirt, so findet man nach Entfernung der K.-S.-L. bei Anwendung derselben Reize, dass die Wirksamkeit der aufsteigenden Ind.-Ströme sehr vergrössert ist; sie kann sogar grösser sein, als die der ab- 
steigenden, obgleich diese auch etwas vergrössert ist, wahrscheinlich durch den geringeren Widerstand des Muskels wegen Befench. tung. Wenn man jetzt die Stärke des Reizes allmäblich vermindert, dauert dieses Verbältniss nicht fort, sondern die Zuckungen verkleinern sich viel schneller bei aufsteigendem Strome als bei absteigendem Strome, sodass, wenn diese letzten noch ziemlich gross, die ersteren nur minimal sind. Wenn dann der Rollenabstand noch mehr vergrössert wird, rufen nur absteigende Ströme Zuckungen hervor. Es zeigt sich dann, dass, wenn man die scheinbaren Erregbarkeiten des untern und oberen Muskelendes dureh die Methode der minimalen Reize vergleicht, die des untern Endes grösser ist; vergleicht man aber die scheinbaren Erregbarkeiten nach der Höbe der durch mittler e Reize hervorgerufenen Zuckungen, so scheint die Erregbarkeit des obern Endes die grössere zu sein, d. b. bei derselhen Vergrösserung des Reizes wächst die Höhe der Zuckungen bei aufsteigendem Strome viel schneller als bei absteigendem Strome. Die Erklärung dieser zuerst überraschenden Erscheinung ergiebt sich, wenn man nicht nur die Stärke des Reizes, sondern auch die Grösse der Belastung variirt. Es folgen die Einzelheiten eines solchen Versuches.

No. V. 11. Juni 1891. Rana temporaria, curarisirter Sartorius, zunächst mit $7 \mathrm{gr}$ belastet, die mit ihrem ganzen Gewicht den Muskel spannten. 5 Daniell'sche Elemente im primären Kreise.

\begin{tabular}{|c|c|c|c|c|c|}
\hline 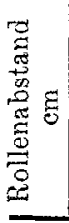 & 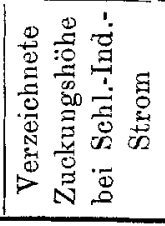 & 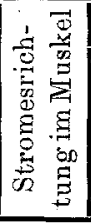 & 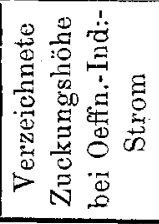 & 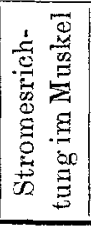 & Bemerkungen \\
\hline $\begin{array}{l}5 \\
5 \\
5 \\
5 \\
7,2 \\
7,2 \\
7,2 \\
7,2\end{array}$ & $\begin{array}{r}30 \\
30 \\
0 \\
0 \\
0 \\
0 \\
18 \\
16\end{array}$ & $\uparrow$ & $\begin{array}{c}27 \\
27 \\
35 \\
36 \\
29 \\
30 \\
2,5 \\
2,5\end{array}$ & $\uparrow$ & $\begin{array}{l}\text { Frischer Muskel an der Luft } \\
\text { gereizt. Der \& S.-I.-Strom wirkt } \\
\text { hier stärker als der } \uparrow \text { Oe.-Z,- } \\
\text { Strom. } \\
\text { Hier wurde das Ende des Rohrs } \\
\text { mit K.-S.-L. gefüllt, so dass das } \\
\text { obere Viertel des Muskels ein- } \\
\text { getancht wurde. }\end{array}$ \\
\hline
\end{tabular}




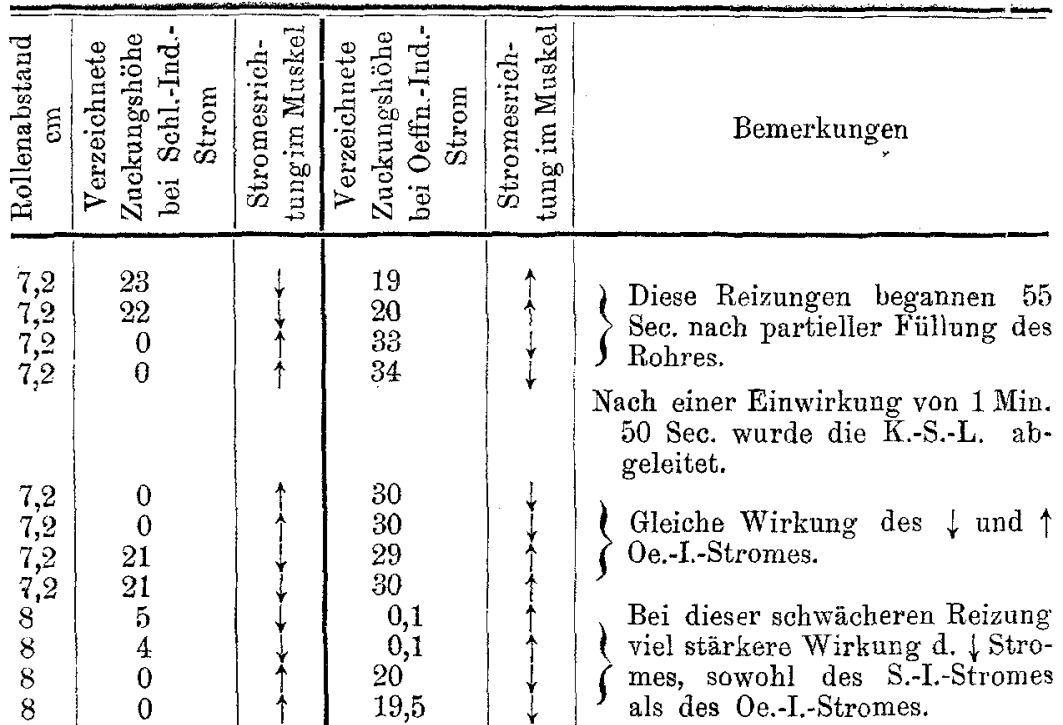

4 Min: 15 Sec. Zwischenzeit.

24,5

24

29

29

23

23

20

19,5

30

30

24

22,5

15,5

15,5

4,5

als des Oe.-I.-Stromes.

4 Min. 15 Sec. Zwischenzeit.

Hier war eine Zwischenzeit von 3 Min.; danach wurded. Muskel mit $3 \mathrm{gr}$ statt $7 \mathrm{gr}$ belastet; die Abscisse stieg $12 \mathrm{~mm}$. 
Die Wirkung d. physiologischen Kochsalzlösung auf quergestreifte Muskeln. 515

\begin{tabular}{|c|c|c|c|c|c|}
\hline 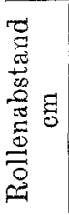 & 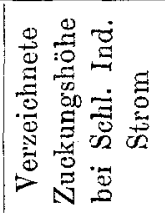 & 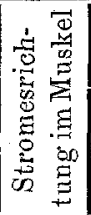 & 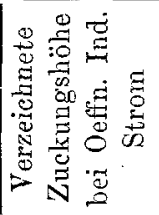 & 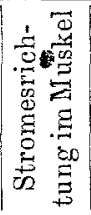 & Bemerkungen. \\
\hline $\begin{array}{l}7,5 \\
7,5 \\
7,5 \\
7,5\end{array}$ & $\begin{array}{c}0 \\
0 \\
17 \\
17,5\end{array}$ & $\begin{array}{l}\uparrow \\
\uparrow \\
\vdots \\
\downarrow\end{array}$ & $\begin{array}{l}34 \\
32 \\
32 \\
35\end{array}$ & $\begin{array}{l}\downarrow \\
\uparrow \\
\uparrow\end{array}$ & $\begin{array}{l}\text { Vergl. die vorhergehenden u. die } \\
\text { folgenden Reizungen bei } 7 \mathrm{gr} \text { Be- } \\
\text { lastung. Beid. kleinen Belastung } \\
\text { sind die Wirkungen der } \uparrow \text { u. } \\
\text { Oe.-I.-Ströme gleich. } \\
\text { Jetzt wurde mit } 7 \mathrm{gr} \text { belastet. Die } \\
\text { Abscisse sank } 13,5 \mathrm{~mm} \text {. }\end{array}$ \\
\hline $\begin{array}{l}7,5 \\
7,5 \\
7,5 \\
7,5\end{array}$ & $\begin{array}{l}9 \\
7 \\
0 \\
0\end{array}$ & $\downarrow$ & $\begin{array}{l}26 \\
25.5 \\
18 \\
18\end{array}$ & $\uparrow$ & $\begin{array}{l}\text { Die Wirkung des } \uparrow \text { Oe.-I.-Stro- } \\
\text { mes wieder grösser als die des } \\
\downarrow \text { Oe.-I.-Stromes. } \\
\text { Jetzt mit } 10 \text { gr belastet. Die Ab- } \\
\text { scisse sank } 7 \mathrm{~mm} \text {. }\end{array}$ \\
\hline $\begin{array}{l}7,5 \\
7,5 \\
7,5 \\
7,5\end{array}$ & $\begin{array}{l}3 \\
3 \\
0 \\
0\end{array}$ & $\downarrow$ & $\begin{array}{l}19 \\
19 \\
13 \\
13\end{array}$ & $\uparrow$ & \\
\hline $\begin{array}{l}7,5 \\
7,5 \\
7,5 \\
7,5\end{array}$ & $\begin{array}{c}0 \\
0 \\
17,5 \\
17\end{array}$ & $\uparrow$ & $\begin{array}{l}32 \\
32 \\
32 \\
31\end{array}$ & $\downarrow$ & $\begin{array}{l}\text { Jetzt wieder mit } 3 \mathrm{gr} \text { belastet. Die } \\
\text { Abscisse stieg wieder. } \\
\left\{\begin{array}{l}\text { Wirkung des } \uparrow \text { u. } \downarrow \text { Oe.-I.-Stro- } \\
\text { mes ist wieder gleich. }\end{array}\right.\end{array}$ \\
\hline
\end{tabular}

Aus diesem Versuche - und das Resultat war. immer constant - ersieht man, dass abgesehen von minimalen und beinahe minimalen Reizungen die s c h e i n bare relative Erregbarkeit der Muskelenden eine Function sowohl der Reizstärke als der Belastungsgrösse ist. Vergrössert man bei constanter Reizstärke die Belastung, so steigt die scheinbare relative Erregbarkeit des Beckenendes; vergrössert man bei constanter Belastungsgrösse den Reiz, so ist dasselbe der Fall. Dies letztere sieht man ganz deutlich ohne Verschiebung der secundären Rolle, wenn die Oeffnungsschläge stark genug sind, um aufsteigend höhere Zuckungen hervorzurufen als absteigend. Beachtet man die Wirkungen der Schliessungsschläge, so sieht man, dass dieselben aufsteigend wirkungslos sind; es gibt nur bei absteigendem Schliessungsschlage Zuckungen (s. Protocoll in vielen Stellen). Bei Schliessungsschlägen scheint das Tibialende, bei Oeffnungsschlägen das Beckenende das erreg. barere. 
Der Einfluss der Belastungsgrösse lässt kaum einen Zweifel, dass der assymmetrische Bau des Sartorius die Ursache der beschriebenen scheinbaren Erregbarkeitsumkehrung ist. Dieser assymmetrische Bau ist nnzweifelhaft die Ursache der von B i e de rm a n $\mathrm{a}$ beschriebeneu und erklärten grössern Wirksamkeit der absteigenden Durchströmung, indem er eine grössere Stromdichte am untern Ende des Muskels bedingt.

Bedenkt man, dass schwache absteigende Ströme zunächst nur am äussersten (untern) Ende des Muskels die nöthige Dichte haben, um die bis dahin reichenden Muskelfasern zu erregen, so erkennt man, dass bei solchen Strömen der Muskel zunächst nur mit einem Theil seiner Fasern in Action tritt, während die übrigen sich bei der Zuckung passiv verhalten. Der aufsteigende Strom versetzt aber, sobald er am Beckenende die zur Erregung nöthige Dichte erreicht, alle Fasern des Muskels gleichzeitig in 'Thätigkeit. Die in beiden Fällen ausgelösten Kräfte sind also sehr verschieden. Ob man beim normalen Sartorius diesen Untersehied direkt beweisen kann, habe ich leider nicht Gelegenheit gehabt zu untersuchen.

Obgleich man leicht sehen kann, dass der assymmetrische Bau des Sartorius die oben beschriebenen Erscheinungen bedingt, so ist es doch nicht leicht, genau anzugeben, in welcher Weise er sie bedingt. Einige Punkte darf ich hier erörter'n.

Offenbar ist bei absteigender Reizung das hierbei allein in Action tretende Faserbiindel gleichsam relativ überlastet. Im Rubezustand wird die Last von a ll e n Fasern des Muskels getragen; bei der Zuckung aber wird diese elastische Stiitze allwälich weggenommen und so contrahirt sich der thätige Muskeltheil, wenn man von der Trägheit des Gewichtes etc. absieht, gegen eine continuirlich zunehmende Kraft.

Wenn man nun das Weber'sche Muskelelasticitätsschema auf die Zuckungen appliciren dürtte, würde die Sache eine sehr einfache Erklärung finden. Wir haben gesehen, dass es unter den gegebenen Versuchsbedingungen bei jeder Belastung eine gewisse Reizstärke gibt, wobei die Inductions-Schläge in beiden Richtungen Zuckungen von gleicher Höhe verursachen. Denken wir uns den Muskel bis zu dieser Höhe contrahirt und nun die Belastung vermehrt: ist die Contraction die Folge eines absteigenden Inductionsschlages, und ist dabei der Muskel nur partiell in Action, so würde 
die Steigerung der Belastung eine grös sere Dehnung bewirken, weil die Last zunächst nur an den activ verkürrten Faserbündeln angreift. Ist aber die Contraction die Folge eines aufsteigenden Inductionsstromes und sind also alle Fasern des Muskels actir verlsürzt, so würde die Steigerung der Belastung eine g e ring e re Dehnung zur Folge baben. Dementsprechend müsste, wenn die Steigerung der Belastung schon vor der Reizung erfolgt und der Reiz derselbe bleibt, der absteigende Strom eine kleinere Verkiirzung bewirken als der aufsteigende, weleher am Beckenende die durch K.-S.-L. gesteigerte Erregbarkeit vorfindet. Es war mir nicht mehr möglich, die geschilderte Abhängigkeit der Reizerfolge von Reizstärke und Belastung am normalen Sartorius (isotonisch bezw. auch isometrisch) zu untersuchen. Die durch K.-S.-L. gesteigerte Erregbarkeit lässt sie offenbar ausgeprägter hervortreten.

Meinen Erfahrungen nach kommen Scheinwidersprüche bei Sartoriusreizversuchen ziemlich häufig vor; z. B. habe ich vielmals die Wirksamkeit von Schliessung'sschlägen bei allmählicher Heranschiebung der secundären Spirale schneller als die der Oeffnungsschläge wachsen gesehen, sodass endlich Schliessungsschläge höhere Zuckungen hervorriefen als Oeffnungsschläge (s. Fig. 2). Vielleicht können einige solcher Scheinwidersprïche ihre Erklärung auf die oben erörterte Weise finden.

IV.

Aus dem Vorhergehenden ersieht man, dass 0,6 procentige K.-S.-L. keineswegs die indifferente Flüssigkeit ist, für die man sie lange gehalten hat. Wir haben sehon in einer Versuchsreihe ihren störenden Einfluss kennen gelernt; es wäre nun zu bedenken, ob ibre Anwendung nicht auch bei andern Untersuchungen Fehler nach sich gezogen hat.

Denkt man an die starken Actionsströme, die ein nur momentan gereizter K.-S.-Muskel geben kann, so ist in Erwägung zu ziehen, ob dieselben nicht eine wichtige Rolle in Untersuchungen über die secundär electromotorischen Erscheinungen der Muskeln gespielt haben, insoweit bei solchen Untersuchungen K.-S.-L. zur Anwendung kam. Dies scheint mir bei der Untersuchung von B i ed erma n $n$ über positive kathodisehe Polarisation bei quergestreiften Muskeln ${ }^{1}$ ) der Fall gewesen zu sein. Wir wissen, dass

1) Sitzungsbericht der Wiener Akad. XCII. Bd. III. Abth. S. 142.

E. Pflüger, Axchiv f. Physiologie. Bd, 54 . 
er die Gewohnheit hatte, bei der Präparation des Sartorins „die blossgelegten Muskeln" „durch einen Strom $3 / 4$ percentiger $\mathrm{NaCl}$ Lösung" abzuspülen und ,sämmtliche bei der Präparation benützten Instrumente beständig mit derselben Lösung benetzt zu erhalten" ${ }^{11}$ ). Auch gewisse Einzelheiten der Resultate lassen sich unter Berück. sichtigung der oben besprochenen Wirkung der K.-S.L. vielleicht einfacher erkłären.

Um diese Frage zu entscheiden, entschloss ich mich, Controlversuche anzustellen. Aeussere Umstände hinderten mich jedoch, dieselben zum Abschluss zu bringen. Doch scheinen meine bisherigen Erfahrungen für meine Auffassung zu sprechen und zu einer Revision der Biedermann'schen Versuche anfzufordern.

Ich ging daran, zu untersuchen, ob sich ein frischer Muskel in Bezug auf "positive kathodische Polarisation" anders verbält, als ein mit K.-S.-L. behandelter. In der That gelang es mir, ein solches verschiedenes Verhalten nachzuweisen, wie aus folgenden Protokollen zu ersehen ist.

Meine Versuchsanordnung war die Biedermann'sche. Eine Bussolelectrode berührte das un tere sehnige Ende des ausgespannten Sartorius, die zweite die Muskelmitte. Die Reizelektroden wurden beiderseits an die Knochen angelegt. Als Reiz (polarisirender Strom) wurde verwendet der Strom von zwei Daniell'schen Eilementen, entweder mit Benützung des Rheochords und Einschaltung von 50 Widerst-Einheiten desselben oder ganz ohne Rheochord.

Die Pfeile zeigen die absteigende (atterminale) oder aufsteigende (abterminale) Richtung des Reizstromes bezw. des Nachstromes im Muskel. Die in der III. Columne verzeichneten Zahlen geben in Theilen der Fernrohrseala die nach Oeffnung des Reizstromes erfolgten Ablenkungen des Magneten aus dem magn. Meridian, in welchen der Magnet vor jeder Durchströ. mung mittels Compensation des Muskelstroms zurückgebracht wurde.

s. 1. z. bedeutet "sehr langsames Zurückgehen" des abgelenkten Mag. neten.

Falls der Magnet nach der ersten Ablenkung schnell in eine entgegen. gesetzte Ablenkung überging, ist seine zweite Grenzlage durch eine unter der ersten Zahl stehende zweite Zahl gegeben. War die Polarisation so nachbaltig, dass ihr Verschwinden nicht abgewartet wurde, so ist in der letzten Columne angegeben, bei welchem noch bestehenden "Polarisations. reste" wieder compensirt wurde. Waren nur wenige Scalentheile zu kompensiren, so ist dies nicht besonders bemerkt.

1) Ebenda LXXXI. Bd. III. Ahth. S. 77. 
Die Wirkung d. physiologischen Kochsalzlösung auf quergestreifte Muskeln. 519

M. R. (Momentreizung) bedeutet eine so kurze Dauer des Reizstromes, wie sie durch möglichst schnelles Hin- und Herwenden der Wippe mit der Hand erreicht werden konnte.

E. S. = Eigenstrom der Boussolelectroden.

M. S. = Muskelstrom.

Rh. W. = Rheochord-Widerstand.

K.-S.-L. = 0,6 procentige Kochsalzlösung.

Nr. VI. 20. Juni 1891. Rana tempor, curarisirt. Sartorius frisch untersucht.

E. S.: $\downarrow 10$ sc. M. S.: $\uparrow 35$ sc.

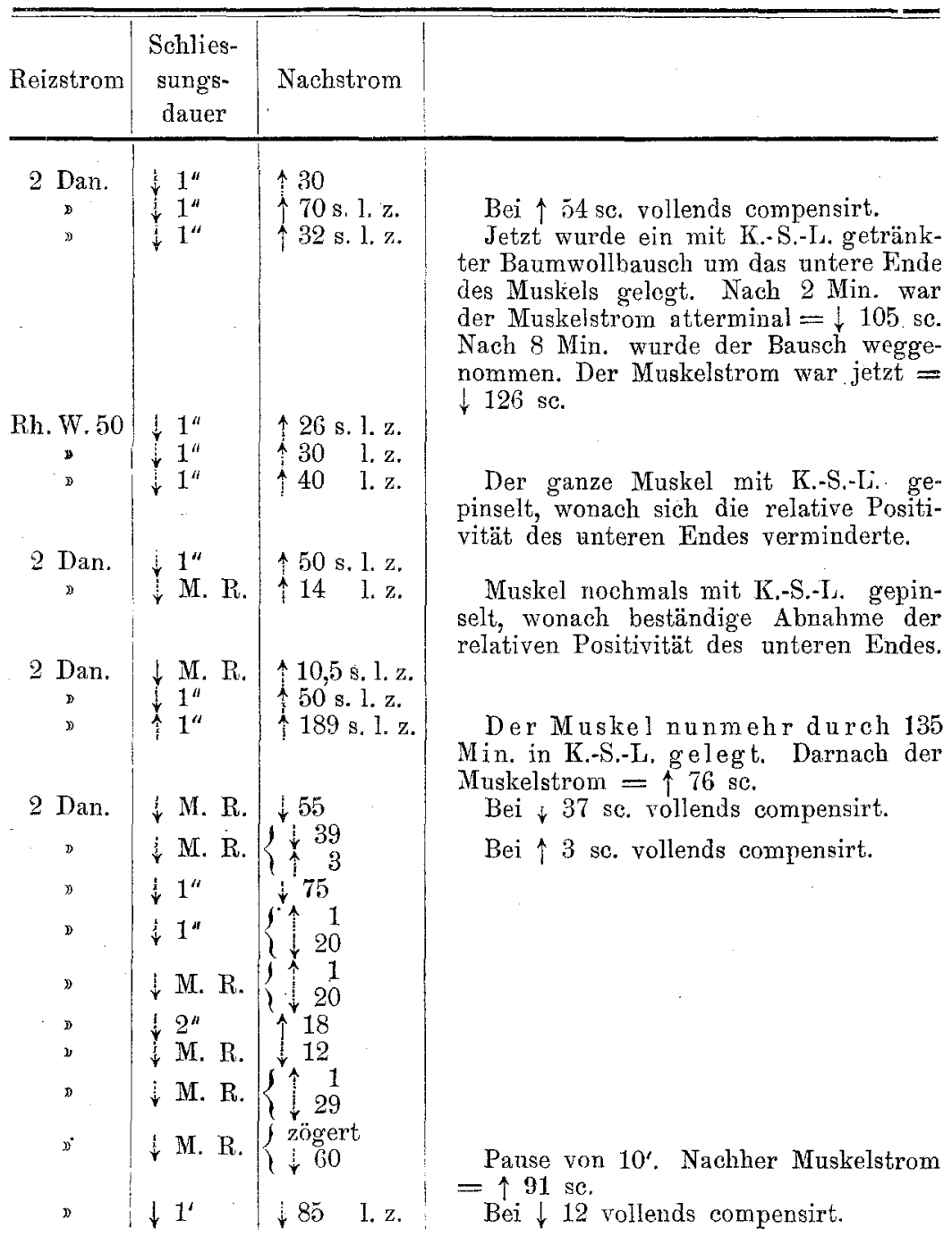




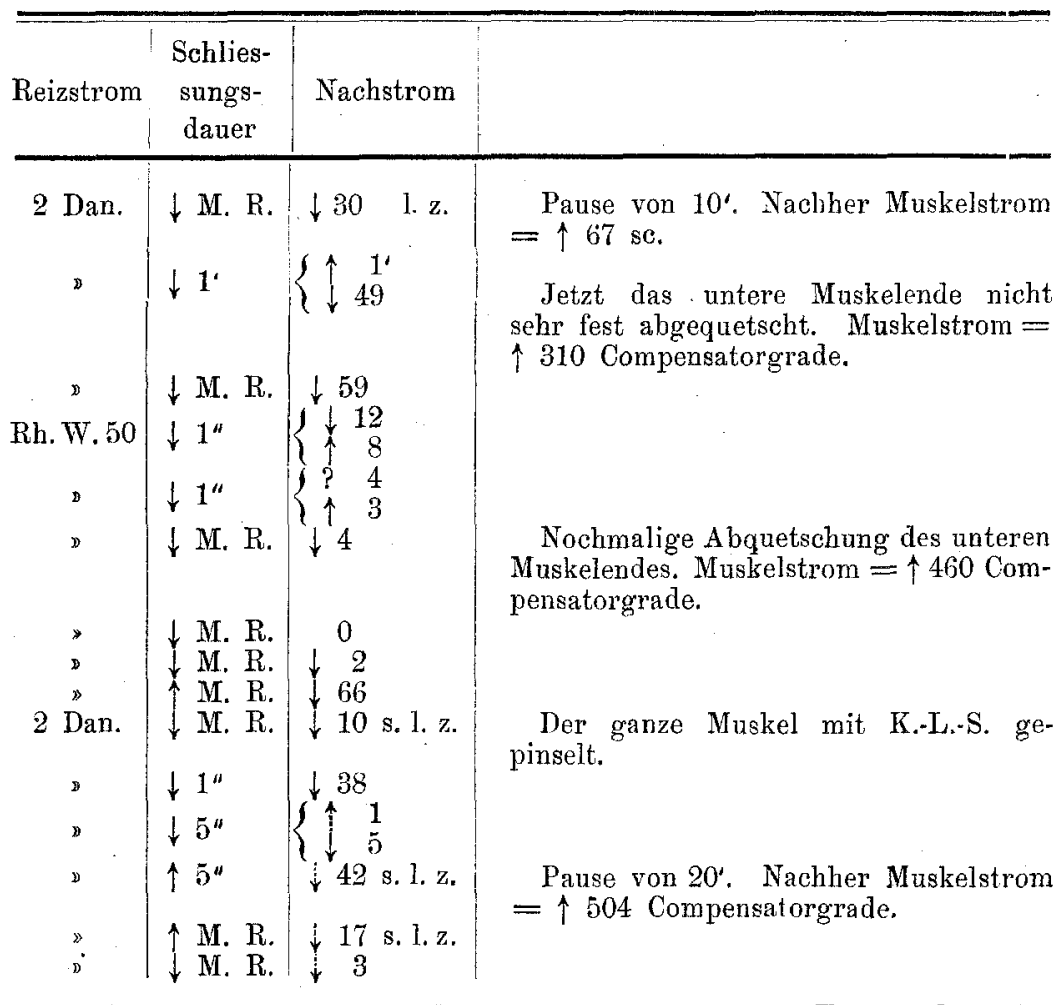

Nr. VII. 20. Juni 1891. Rana temporar. curarisirt. Erster Sartorius frisch untersucht. = M. S. 20 sc.

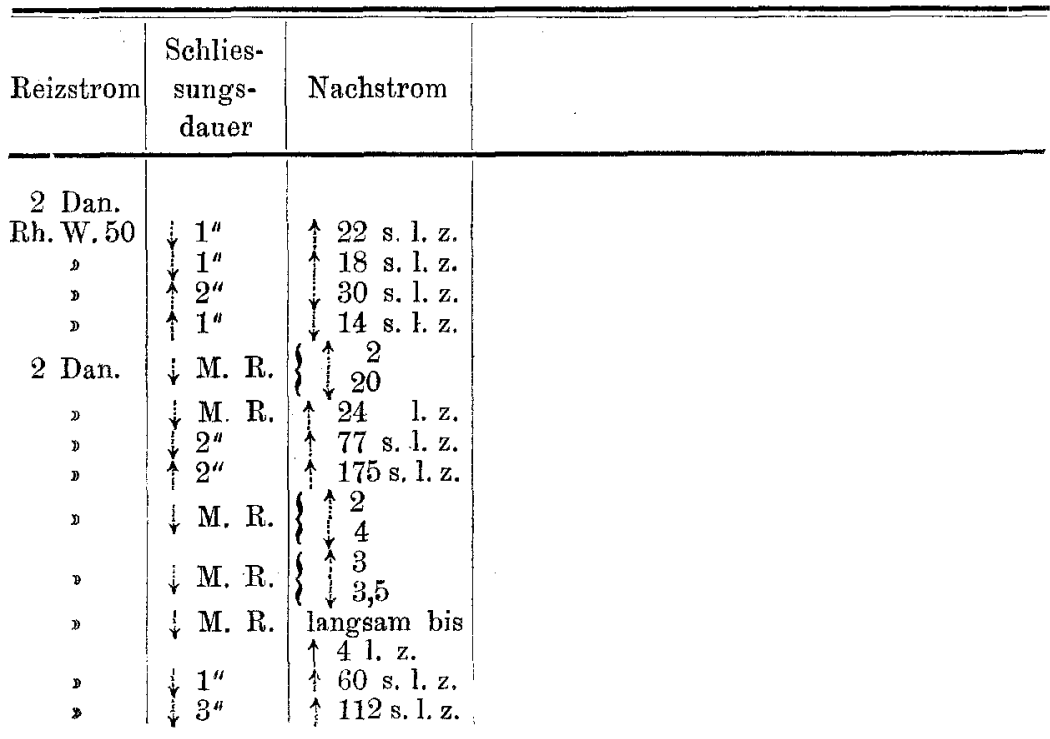


Die Wirkung d. physiologischen Kochsalzlösung auf quergestreifte Muskeln. 521

Zweiter Sartorius (dessen oberes Ende mittels Fadenumschnürung fixirt war) nach Einlegung in K.-S.-L. durch 70 Minuten.

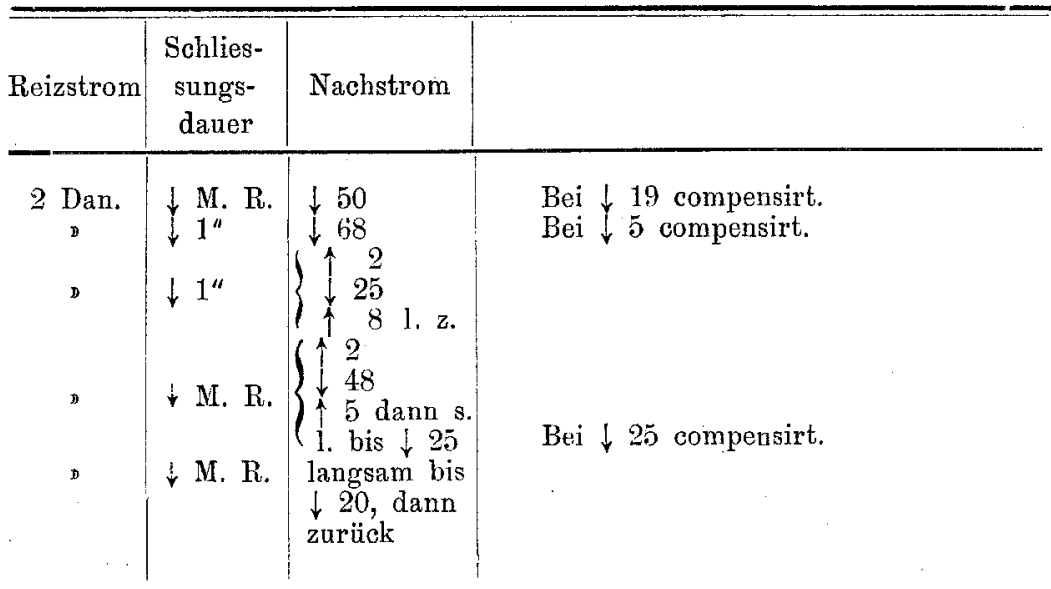

Wie man siebt, zeigte sich, abgesehen von geringfiugigen $A b-$ weichungen (Nr. VII), die positive kathodische Polarisation erst nach Behandlung der Muskeln mit K.-S.-L. Bei der relativ langen Nachdauer der Contractionen eines Kochsalz-Muskels ist zu bedenken, dass die durch Schliessung des Reizstromes bewirkte Erregung des Muskels die schnell folgende Wiederöffnung überdauern kann, sodass, auch wenn die Oeffnung nicht abermals erregend wirkt, der ganze Muskel noch in Erregung sein kann, während bereits der Nachstrom von ihm abgeleitet wird. Ist im Augenblicke der Schliessung des Boussolkreises der ganze Muskel noch im erregten Zastande, so kann leicht der Fall eintreten, dass die der Erregung entsprechende relative Negativität der Muskelsubstanz an der Muskelmitte einen grössern negativen Zuwachs bedingt als am Muskelende. Dies aber müsste einen „positiven kathodischen Polarisationsstrom" ergeben.

Dies wäre besonders dann möglich, wenn das kathodische Muskelende zuvor abgetödtet worden ist, und daher ein starker abterminaler Muskelstrom besteht, welchenfalls dann, sofern bei Sehliessung des Boussolkreises die Erregung des Gesammtmuskels noch andauert, die negative Schwankung des Muskelstromes als positive Polarisation erscheinen würde.

Allerdings pflegt unter gewöhnlichen Umständen ein durch das abgetödtete Ende des Muskels austretender Strom bei der Schliessung keine Erregung au bewirken, aber dies gilt doch nur 
dann, wenn der Strom nicht zu stark und die Erregbarkeit des Muskels nicht künstlich gesteigert ist. Am kathodischen Muskelende können sich bei Anwendung stärkerer Ströme immerhin noch innerhalb der erregbaren Substanz wirksame Austrittsstellen des Stromes finden, z. B. wenn hier noch etwas Flïssigkeit auf der Muskeloberfläche haftet, sodass der Strom nicht ausschliesslich durch todte Substanz den Muskel verlässt. Wurde insbesonders die Abtödtung des kathodischen Muskelendes durch concentrirte $\mathrm{NaCl}$, oder $\mathrm{Na}_{2} \mathrm{CO}_{3}$-Lösung bewirkt, so könnten diese Stellen hochgradig erregbar sein. Diesenfalls wird also das „polare Versagen“ mehr oder minder unvollständig sein. Dazu kommt, dass auch die an der Muskelmitte anliegende Boussolelectrode wäbrend der Schliessung des Reizstromes das Ausbiegen eines kurzen Stromzweiges in die Spitze der Electrode und so die Bildung einer kathodisehen Stelle gestattet; was bei starken Strömen (2 Daniell) und gesteigerter Erregbarkeit ins Gewicht fallen kann.

Es ist also nöthig, durch Inspektion des Muskels während des Versuches seine Contractionen, die sich ja auch am ausgespannten Muskel mehr oder weniger verrathen, möglichst sorgfältig zu controliren. In der That habe ich Kochsalzmuskeln, während sie einen positiven kathodisehen Polarisationsstrom gaben, in nachdanernder Contraction gesehen.

Analoge Betrachtungen lassen sich bezïglich der von Biedermann beobachteten negativen anodischen Polarisation anstellen.

Auch die Thatsache, dass längere Schliessungsdauer die positive kathodische Polarisation hindert, liesse sich aus unserem Gesichtspunkte leicht erklären, weil unter solchen Umständen eine verlängerte Schliessungserregung des Gesammtmuskels Zeit hat, vollständig abzuklingen, ehe der Boussolkreis geschlossen wird. Auch wäre leicht verständlich, warum die Behandlung des unversehrten Muskelendes mit schwacher $\mathrm{Na}_{2} \mathrm{CO}_{3}$-Lösung die positive kathodische Polarisation nicht fördert. Denn hierdurch wird nur die Nachdauer der bei der Schliessung oder Oeffnung bewirkten Erregung jener Muskelpartie und also, wie Biedermann selbst angibt, die negative kathodische und die positive anodische Polarisation an diesem Muskelende gefördert.

Anderseits muss ich betonen, dass ich ebenfalls (s. Protokoll Nr. VII) positive kathodische Polarisation am frischen Muskel gesehen habe, und dasselbe mitunter auch bei Muskeln, die mit 
$\mathrm{CaSO}_{4}$-haltiger K.-S.-L. längere Zeit behandelt worden waren. Die Nachströme waren jedoch im Vergleich mit denen bei Kochsalzmuskeln nur klein.

Jedenfalls müssten, um eindeutige Ergebnisse zu erhalten, die mechanischen Reizerfolge gleichzeitig möglichst genau controlirt werden, wobei vielleicht auch das Fick'sche isometrische Verfahrèn Dienste leisten könnte.

Die vorhergehende Erörterung bezieht sich auf Biedermann's Nachweis der positiv kathodischen Polarisation bei normalen Muskeln. Es braucht kaum erwähnt zu werden, dass die angestellten Erwä. gungen in viel höherem Grade bei seinen Versuchen mit partiell veratrinisirten Maskeln in Betracht kommen. Fand es ja Biedermann zweckmässig, die Polarisationsversuche nicht unmittelbar nach dem Entfernen des Muskels aus der Giftlösung, sondern erst nach längerem Verweilen ( $1 / 4$ Stunde und mehr) des letzteren in physiologischer Kochsalzlösung vorzunehmen. Dieses Verfahren ermöglichte nicht nur eine Einwirkung der K.-S. L., sondern vielleicht auch eine durch Diffusion des Giftes hervorgerufene schwache Veratrinisirung des ganzen Muskels.

Die von Biedermann am partiell contrahirten Froschmuskel auf galvanometrischem Wege beigebrachten Beweise für das Bestehen einer bei Oeffnung des Reizstromes an der Kathode eintretenden Hemmung des bestehenden Erregungszustandes, und für die gleichzeitige Entwicklung relativer Positivität des kathodischen Muskelendes sind also nicht einwurfsfrei, wenngleich die auf graphischen Wege von ihm gewonnenen Ergebnisse, sowie seine Erfahrungen am tonisch contrahirten Muschelmuskel dafür sprechen.

\section{Erläuterung za der Tafel.}

Sämmtliche Curven stammen von Sartoriusmuskeln, die mit Ausnahme von Fig. 2 curarisirt waren. Als primäre Stromquelle dienten bei Fig. 1 und 2 ein Daniell'sches Element, bei den anderen zwei. Im primären Kreise fand sich ein Quecksilberschlüssel, im secundären Kreise eine Pohl'sche Wippe und ein Vorreiberschlüssel. Die Schläge wurden von den Knochen. stümpfen aus durch den ganzen Muskel geschickt. Die wahre Spannung des Muskels betrug immer nur 0,35 der Belastung. Der Zeitmerker merkt Secunden. Bei sämmtlichen Figuren mit Ausnahme von Fig. 1 und 2 ist der Moment der Schliessung und Oeffinung des primären Stromes mittels eines Electromagnets verzeichnet. 
524 F. S. Locke: Die Wirkung der physiologischen Kochsalzlösung etc.

Die Pfeile bedeuten die Stromesrichtung im Muskel; die Kreuze Wendung der Wippe; die Zahlen den Rollenabstand in Centimetern.

Fig. 1 und 2 sind anf $2 / 3$ ihrer wirklichen Grösse durch Photographie reducirt.

Fig. 1 Belastung von $20 \mathrm{gr}$. Der Muskel nach Eintauchung durch eine halbe Stunde in K.-S.-L. wurde bei allmählicher Verminderung des Rollenabstandes gereizt. Die abgebildeten Curven entstanden bei $0,3 \mathrm{~cm}$ Rollenabstaand. Man sieht die Wirkung eines aufsteigenden Schliessungsschlages und eines absteigenden Oeffnungsschlages.

Fig. 2. Belastung von $7 \mathrm{gr}$. Der frische, nicht curarisirte Muskel war sehr viel bei allen Reizstärken gereizt worden, und gab nur einfache Zuckungen. Nach Eintauchung für eine halbe Stunde in K.-S.-L. wurde er wieder wie bei Fig. 1 gereizt; die abgebildeten Curven entstanden bei 0 Rollenabstand. Die Stromesrichtung war leider nicht angemerkt.

Fig. 3. Frischer bei O Rollenabstand gereizter Muskel. Es zeigt sich ein kleiner Verkürzungsrückstand, besonders bei Oeffnungsschlägen.

Fig. 4. Derselbe Muskel wurde in K.-S.-L. durch 10 Min. gelegt, und nachher bei allen Reizstärken vielmals gereizt, wobei er die Kochsalzwirkung ganz gut zeigte. Dann wurde er noch einmal für 17 Min. in K.-S.-L..gelegt. Jetzt schrieb er die abgebildeten Curven. Die Contraction ist sehr verlängert, und trat bei diesen Rollenabständen nur bei absteigenden Oeffnungsschlägen ein.

Fig. 5. Jetzt wurde derselbe Muskel sofort in $\mathrm{CaSO}_{4}$-hältige K.-S.-L. durch 5 Min. gelegt und dann bei allmählicher Verminderung des Rollenabstandes von $8,5 \mathrm{~cm}$ bis 0 gereizt. Die abgebildeten, bei einem Rollenabstande von $3,5 \mathrm{~cm}$ geschriebenen Curven sind besonders typisch. Es zeigt sich ein kleiner Verkürzungsrückstand.

Fig. 6. Nachdem der Muskel während einer Stunde in der feuchten Kammer geblieben war, wurde sein normales Verhalten bewiesen, und or sodann in chemisch reine K.-S.-L. durch 20 Min. gelegt. Nachher wurde der Muskel bei allmählicher Verminderung des Rollenabstandes gereizt. Man sieht den Einfiuss der Stromstärke, Stromesrichtung, und den Unterschied in der Wirkung von Schliessungs- und Oeffnungsschlägen.

Fig. 7. Die abgebildete Curve wurde von demselben Muskel gleich nach denjenigen der Fig. 7 geschrieben. Die Trommelgeschwindigkeit ist, jetzt viel grösser, um den zeitlichen Verlauf der Contraction zu zeigen.

Fig. 8. Der Muskel blieb nach einigen Reizungen während 2 Stunden in K.-S.-L. Nachher wurde er bei Rollenabständen von $2.5,7$ und $2.5 \mathrm{~cm}$ gereizt, und sodann die abgebildeten Curven bei O-Rollenabstand und ohne Umkehrung der Stromesrichtung geschrieben, um die Verminderung dex Höhe der Contractionen bei fortgesetzter Reizung zu zeigen. Nachher wurde der Muskel in K.-S.-L. durch 25 Min. eingetaucht, und sodann unter denselben Bedingungen abermals gereizt:

Fig. 9. Die Contractionshöhe ist jetzt vergrössert. Man sieht die erholende Wirkung der Kochsalzlösung. 